\title{
ASTROMETRY AND ORBITS OF ASTEROIDS AND COMETS
}

\author{
Brian G. Marsden \\ Harvard-Smithsonian Center for Astrophysics \\ 60 Garden Street \\ Cambridge, MA 02138 \\ U.S.A.
}

\begin{abstract}
Astronomers have been consciously making positional observations of comets since the sixteenth century, and rough information on bright comets is available from social records collected in the Far East, the Middle East and Europe over the previous two millennia. Attempts at representing the observations with orbits also date back to the sixteenth century, well before the earliest computations were made on the basis of gravitational theory. Improvement in the accuracy of cometary astrometric data was slow to develop following the invention of the telescope and, a few decades later, the micrometer, for this had to await the availability of Flamsteed's star catalogue and the understanding brought about by Bradley's discovery of aberration and nutation. In spite of important contributions by some of the most celebrated scientists of the eighteenth century, orbit computation remained largely a process of trial and error until the discovery of the first asteroids and the rise of Gauss and his followers. By the 1880 s the availability of a dense star catalogue like the AGK1 was allowing micrometric observations of comets and asteroids often to achieve an accuracy of 2 to $3 \mathrm{arcsec}$, and the subsequent introduction of photographic astrometry brought convenience but relatively little additional improvement in accuracy. The use nowadays of charge-coupled devices clearly has the potential for a further increase in accuracy, even for diffuse objects like comets, but for the moment the accuracy tends generally to be limited by the inadequacy of the available star catalogues. Modern orbit determination routinely includes the use of sophisticated techniques to identify isolated observations of the same asteroid at different oppositions and in the case of a comet the examination of the nongravitational effects that can strongly influence the object's motion.
\end{abstract}

Although computerized databases of orbital information on comets and asteroids were already being developed in the 1960 s, access to such databases was largely limited unti] versions of the IAU Minor Planet Center's catalogues of orbits of comets and of unnumbered minor planets were issued on MS-DOS diskettes in 1986. As early as January 1984, however, subscribers to the Computer Service operated by the Minor Planet Center and the IAU Central Bureau for Astronomical Telegrams could dial up to obtain orbital data on individual objects from databases that are generally updated monthly, in concert with the publication of the batches of printed Minor Planet Circulars. The complete orbital databases maintained by the Minor Planet Center include files of 'original' and 'future' cometary orbits, cometary nongravitational parameters, osculating elements of all the numbered and multiple-opposition unnumbered asteroids at intervals of 200 days since 1900 and 1960, respectively, and cross-references giving all the provisional designations 
that refer to the same object. Beginning in 1991, the Leningrad Institute for Theoretical Astronomy, which is responsible for the publication of orbits and ephemerides of the numbered asteroids, is also issuing the data and supporting computer programs on an MSDOS diskette.

Since orbital elements are derived from astrometric observations, it is sufficient for many purposes to regard the observations as adequately represented by the orbital elements and hence that the orbits are in some sense highly reduced "observations" of the comets and asteroids concerned. In the absence of detailed information on how well an orbit actually represents the observed positions, one would have to regard these orbital "observations" as fixed entities, there being no way to render them more accurate in the future. But new astrometric observations do allow orbit computations to be improved, and it is therefore appropriate to consider a set of orbits as comprising a database, rather than an "archive". The latter term more suitably describes the file of astrometric observations from which the orbits are computed.

The archive of astrometric observations maintained by the Minor Planet Center began with the merging of sets of observations of asteroids collected during the tenure of Paul Herget as director and observations of comets collected long ago by the present writer. The original intent was that the asteroidal data would be complete back to 1939 and the cometary data back to 1964 , although many earlier observations were also included. The data consist of the object's designation, the UT time of observation, the 1950.0 right ascension and declination, a five-character reference to the publication, and a three-figure code to indicate the place of observation; if available, the magnitude is also supplied, and there is also an indication if the originally published designation of an asteroid subsequently had to be corrected. As with the orbits, the astrometric archive is updated when the monthly batches of Minor Planet Circulars are prepared. Copies of the complete archive, which currently contains more than 600000 observations, are issued on magnetic tape every three years or so, and the monthly additions (and information about deletions and other adjustments) are issued on MS-DOS diskettes.

Soon after the Minor Planet Center moved from Cincinnati to Cambridge, the archive was extended to include at least some observations of every asteroid for which a new-style provisional designation was supplied, which generally means for all asteroids discovered since 1925 . The vast majority of these additional observations were published in the Circulars of the Astronomisches Rechen-Institut. The distribution of these Circulars was extraordinarily limited, and the high sulphuric acid content of the mimeographed pages (some of the material having originally been handwritten) has guaranteed that virtually no sets remain intact. Since the only records of many of these old observations are literally crumbling away, efforts are currently therefore being made to include all the data in these Circulars into the archive, and many pre-1925 observations from other publications are being incorporated too. The problem is greatly compounded by the existence of many thousands of gross errors (involving designations, months of observation, hours of right ascension, the sign of the declination, etc.) in the old data. Gareth Williams, Associate Director of the Minor Planet Center, has been putting a tremendous effort into attempting to correct these errors. In accordance with the recommendation of IAU Commission 20 that the J2000.0 system be introduced in 1992 for work on comets and asteroids, the Minor Planet Center is also in the process of attempting to convert the observations, a task that is complicated in some instances by previous failure to note whether allowance for the elliptic aberration terms was made or not. 
Although it may therefore seem reasonable to speak of the astrometric observations as forming an archive, it is nonetheless clear that the information in the file is in fact far removed from the original material, even for the observations not affected by gross errors. In this connection, it is useful to remark in particular on the manner in which the "Astronomische Nachrichten" and other leading journals used to publish micrometric observations. The standard formulation was to give the time of observation in local mean time, the offset in R.A. and Decl. from the reference star, the object's apparent position, the reference star's position with respect to the mean equinox of the beginning of the year, and the reduction from mean to apparent place. Apart from the redundancy often helpful in tracking down gross errors, there therefore exists the very real possibility of improving the object's position with the help of a better position for the reference star. With the help of an orbit determination, it is frequently also possible to detect and correct an error in the identification of the reference star. The only real problem with micrometric observations is then that the time may have been incorrectly recorded, and even then, a little detective work can uncover gross errors.

Of course, accurate photographic astrometry requires three, or preferably more, reference stars, and the need for economy has usually precluded the possibility of publishing information about them. In some publications three reference stars might be briefly identified, and from the available positions it was therefore possible to deduce the "dependences", i.e., the essentially linear combination of the star positions that yields the object position. Particularly if there were more reference stars, the dependences might be specifically stated. In either case, the position of the asteroid or comet could, at least in principle, be corrected if improved positions became available for the reference stars. However, as computing capabilities have improved, the dependence method has largely fallen into disuse. The publication instead of the rectangular coordinates of object and reference stars actually measured on the plate would, of course, have allowed new solutions by plate constants, even to the extent of including terms of second and higher order that may have been ignored by the original measurer.

In any case, again in principle, there would presumably always be the possibility of remeasuring the plates. Although the digitization of a wide-field plate is currently a rather time-consuming process, this situation will undoubtedly improve in the future. In the mean time--and with the recognition that there is deterioration with time--a photographic plate is clearly itself an excellent storage device and archive for the positions of the various images contained on it. Practitioners of other fields of astronomy have obviously come to the same conclusion. An important consideration for the comet/asteroid astronomer--if perhaps not for other astronomers--is the continued existence and preservation of the log books that give the precise times of the beginning and end of each exposure. In fact, the existence of computerized subarchives with the mid-times of observation and the centers, sizes and limiting magnitudes of the exposed fields is also of value, the systematic computation of "residuals" of each plate center from the expected position of each object then allowing all the plates likely to contain an image to be flagged.

The CCD is now rapidly superseding the photographic plate as the medium of choice, particularly for small-field astrometry with modern reflectors. The convenient availability of the Space Telescope Science Institute's "Guide Star Catalogue" has proven to be a great boon, at least to those observers with fields of view on the order of 10-15 arcmin. Although the number of CCD comet/asteroid astrometrists is still little more than half a dozen, it is clear that most of these observers entered the CCD era rather precipitously, and 
at least initially, little thought was given to the permanent preservation of the raw data. Timings are essentially error free, and the "plate reductions" are fully automated. Nevertheless, the first edition of the "Guide Star Catalogue" is afflicted by significant errors near the corners of the Schmidt plates from which it was prepared, there are difficulties arising from the use of the 1960s-vintage SAO Catalogue for the reduction of these 1980s-vintage Schmidt plates, and as time goes by, the absence of proper motions will progressively become a problem. It should also be considered that the data may be useful for photometric purposes. Given the intrinsic accuracy and linearity of the CCD process, it stands to reason that future re-reductions of the data would be desirable --yet the magnetic tapes with the only record of the original images have in many instances been reused.

All the CCD astrometrists are aware of the problem of destruction of raw data, however, and most of them are now starting to do something about it. In any case, it seems that the actual rectangular measurements of the objects and reference stars have been saved, if only on paper. The present solutions seem to go along two routes. One, adopted at the Oak Ridge Observatory in Massachusetts, is to save only those sections of the CCD frames that contain the objects of interest. A monthly census allows the archiving on standard, 2400foot, 9-track tapes of $33 \times 33$ square pixels around each object or reference star, thereby reducing the volume to some 2 to 3 percent of the original. Thought is being given to making an even further reduction. But although it is significant to note that a back-up copy of the Oak Ridge tapes is maintained at a separate site, severe reduction of the data of course means that, unless one can initially identify everything of interest in the field, information of potential interest to future astronomers is still lost.

The other solution is therefore to try to save everything, something one will certainly want to do when CCD astrometry has extended to wider fields, perhaps with hundreds or more asteroids on each record, At least three of the present, narrow-field CCD astrometrists are attempting to store all their raw image data, and they are doing this on Exabyte 8-mm tapes, which occupy much less storage space than the full-size tapes. This may in fact be the best solution at the present time. Undoubtedly, it will be found that a small fraction (perhaps even as much as 10 percent) of these tapes will not be readable in the future, and future technicians will be faced with the task of ensuring that the information is always rerecorded on new media and in formats that will become standard in the future. In this connection, one already thinks of optical disks, but complexities in writing information to them, and particularly in rewriting information to them, do not currently make them a viable option. 